\title{
High level of pattern glare in major depressive disorder
}

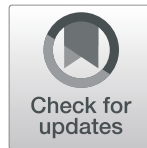

\author{
Xiongwei Qi', Huanhuan Fan', Xiao Yang ${ }^{1}$, Yayun Chen', Wei Deng ${ }^{1,2}$, Wanjun Guo ${ }^{1,2}$, Qiang Wang ${ }^{1,2}$, Eric Chen ${ }^{3}$, \\ Tao $\mathrm{Li}^{1,2}$ and Xiaohong $\mathrm{Ma}^{1,2^{*}}$ (D)
}

\begin{abstract}
Background: Visual deficits have been reported in abundance by recent studies on major depressive disorder. Pattern glare manifests as visual distortions, such as the symptoms of headache, glare, eyestrain, illusions of shapes, colors, and motion when viewing repetitive striped patterns, of which some can be observed in major depressive disorder. Inspired by what mentioned, the present study aims to explore whether there exists association between pattern glare and major depressive disorder and further attempts to explore possible clinical diagnostic value of pattern glare in major depressive disorder.

Methods: Twenty-four patients diagnosed with major depressive disorder (MDDs group) were compared with 30 age-, gender- and education level-matched healthy control subjects (HCs group) on their visual stress with blackand-white gratings of different spatial frequencies-0.3 (low-SF), 2.3 (mid-SF), and 9.4 (high-SF) cycles per degree (c/deg)-which was named pattern glare test. The MDDs group divided into first episode medication-free group (FMDD) and recurrent medicated group (rMDD), comparisons of pattern glare scores (PGS) were performed within the MDDs group. We used Pearson and Spearman analysis to explore the relationship between some clinical indexes and pattern glare scores. ROC (receiver operating characteristic) curve was used to evaluate whether pattern glare test was able to discriminate patients and healthy controls.
\end{abstract}

Results: The mid-SF pattern glare score significantly elevated in patients with major depressive disorder compared to control subjects. No differences of pattern glare scores were found between fMDD and rMDD. A significant negative correlation between mid-high difference and age in HCs group was found. There were no correlations between other variables and pattern glare scores. The mid-SF score has limited value in the diagnosis of major depressive disorder.

Conclusions: We observed an increased level of pattern glare in patients with major depressive disorder, reflecting the existence of cortical hyper-excitability in major depressive disorder. The mid-SF score may have a value in understanding cortical excitability in major depressive disorder.

Keywords: Major depressive disorder, Pattern glare, Visual deficits, Cortical hyper-excitability

\section{Background}

Major depressive disorder (MDD), affecting thousands of millions of people all around the world [1], is one of the most debilitating disease with the symptoms of low mood, declined interests and impaired cognition [2], along with somatic ones, such as headache [3-7] and insomnia [7]. Major depressive disorder was reported to

\footnotetext{
* Correspondence: maxiaohong@scu.edu.cn

'Psychiatric Laboratory and Mental Health Center, West China Hospital of Sichuan University, Chengdu, Sichuan 610064, People's Republic of China ${ }^{2}$ West China Brain Research Center, West China Hospital of Sichuan University, Chengdu, China

Full list of author information is available at the end of the article
}

be one of the five leading causes of years lived with disability (YLDs) in 2016 globally, resulting in 34.1 million of total YLDs [8].

Several lines of evidences show that there exist visual abnormities in patients with major depressive disorder. Photophobia, perceived dimness, anomalous preattentive processing of visual information and self-reported visual function loss were found to exists in patients with major depressive disorder [9-14]. Using the CogState battery (CSB) Chinese language version, a sensitive cognitive assessment instrument, an impairment in the visual, working, and verbal memory was found in first-episode, drug-

(c) The Author(s). 2019 Open Access This article is distributed under the terms of the Creative Commons Attribution 4.0 International License (http://creativecommons.org/licenses/by/4.0/), which permits unrestricted use, distribution, and reproduction in any medium, provided you give appropriate credit to the original author(s) and the source, provide a link to the Creative Commons license, and indicate if changes were made. The Creative Commons Public Domain Dedication waiver (http://creativecommons.org/publicdomain/zero/1.0/) applies to the data made available in this article, unless otherwise stated. 
free MDD patients in a Chinese population [15]. Longer visual search time was needed when detecting a target calling for difficult attentive search strategy in patients with MDDs than in controls [16]. Retinal contrast gain, visual contrast sensitivity, and visually evoked potentials (VEPs) amplitudes were found to decrease significantly in patients with MDD, by utilizing the method of pattern electroretinogram (PERG), subjective visual contrast test and VEPs recordings [17-20]. The normalization of decreased contrast gain after anti-depressant treatment was also reported, the therapies being varied markedly [21]. Developmentally and anatomically, the retina is thought to be an extension of the CNS and thus much of knowledge and discoveries obtained from eye research could be applied to the CNS [22].

Apart from the methods of PERG recording, VEPs recording and subjective visual contrast test, pattern glare test is another tool to explore vision abnormities. Some people can perceive visual perceptual distortions and discomfort, manifesting as the symptoms of eyestrain, headaches and glare, as well as illusions of shapes, colors, and motion, when viewing repetitive striped patterns $[23,24]$. The extent of these experiences varies according to the character of the pattern and individual susceptibility [25]. High-contrast striped patterns, with spatial frequency around 3 cycles/degree and with equal width and spacing, tend to generate maximal effect [25]. The phenomenon depicted above has been named 'pattern glare' [26]. The term 'Meares-Irlen syndrome' or 'visual stress' was also used to describe the symptoms generated by pattern glare [27]. The neural mechanism underlying pattern glare is widely considered as cortical origin, that is, cortical hyper-excitability or poor cortical inhibition generated by deficiency of inhibitory mechanisms that are insufficient to contain the overexcited conditions [25, 27-33]. An increase of blood oxygenation in the visual cortex, evidenced by the fMRI BOLD signal, was found when subjects responded to squarewave gratings [34]. Jason J. Braithwaite and colleagues concluded that heightened levels of pattern glare can reflect increasement of cortical hyperexcitability associated with some possible abnormal experiences in some nonclinical populations [35]. Pattern glare has been assessed using The Wilkins and Evans Pattern Glare Test, which was first performed by Wilkins and Evans to identify individuals susceptible to visual stress by means of pattern related questionnaire, by counting scores for the number of visual illusions and discomfort induced by viewing three high-contrast gratings of spatial frequencies $0.5 \mathrm{cy}-$ cles per degree (cpd), $3 \mathrm{cpd}$, and $12 \mathrm{cpd}$ [27]. The application of pattern glare test in a great variety of neurological conditions, such as synaesthesia, autism, myalgic encephalomyelitis, multiple sclerosis, stroke, and reading ensued [36-43]. To the best of our knowledge, there is no paper reporting the association between major depressive disorder and pattern glare.

Inspired by the above-mentioned evidences that patients with major depressive disorder display various vision abnormities and pattern glare-related symptoms, such as headache and photophobia, we presume high level of pattern glare in the disease, which is assessed by using the pattern glare test. Meanwhile, the current way to diagnose major depressive disorder, depending mainly on the self-reporting of symptoms and mental evaluation, accompanies with the chance of misdiagnosis, which calls for a reliable biomarker with good accuracy for identifying the disease [44]. We attempted to explore possible diagnostic value of pattern glare test, as a potential biomarker, in major depressive disorder.

\section{Methods}

\section{Participants}

We recruited 24 patients diagnosed with major depressive disorder (MDDs group) and 30 age-, gender- and education level-matched healthy controls (HCs group) for the study; all the participants were right-handed. The study was conducted in accordance with the Declaration of Helsinki and was approved by the West China Hospital of Sichuan University ethics committee. All the 54 subjects provided written informed consent.

The 24 MDDs were recruited at the Mental Health Centre of West China Hospital, Sichuan University, People's Republic of China. Inclusion criteria of MDDs group were the current diagnosis of a major depressive episode according to DSM-IV (Diagnostic and Statistical Manual of Mental Disorders, fourth edition) - Patient Version (SCID-P) criteria classified as either MDD with single episode or recurrent episode [45]. Exclusion criteria were as follows: [1] were younger than 18 years or older than 60 years, pregnant or breast feeding, or mentally retarded [2]; had major physical diseases, such as traumatic brain injury, encephalitis, epilepsy, or endocrine disease [3]; had a history of psychotic symptoms during the whole disease duration [4]; had other axis I disorders, such as generalized anxiety disorder, obsessive-compulsive disorder, or drug or alcohol abuse [5]; had other disease that can induce pattern glare, such as dyslexic, autistic, multiple sclerosis, migraine, stroke [6]; had any difficulties in completing the test, such as suffer from eye disease and impaired visual acuity.

The 30 healthy controls (HCs) were recruited locally by advertisement and were screened for a lifetime history or current depressive episode and other neuropsychiatric illness using the Structured Clinical Interview (SCID-NP) in the DSM-IV Non-Patient Edition [46]. We also excluded the controls whose firstdegree relatives had any psychiatric illness or had a current or history of depression or other axis I disorders 
and assured that all the HCs had no other diseases which can induce pattern glare.

All participants were assessed immediately after recruitment in our study. All subjects were confirmed to be within the normal vision acuity utilizing the Snellen chart [47-49]. The severity of diseases was assessed using a 17-item Hamilton Depression Rating Scale (HAMD). Ten of the 24 depressive patients suffered from first episode and were all medication-free, while the other 14 ones from recurrent episodes and medicated.

\section{Pattern glare test}

Pattern glare test has been regarded as a simple but established way to measure visual stress which reflects cortical hyperexcitability $[25,35]$. The pattern glare test consists of three patterns with different spatial frequency, all of which were presented at $40 \mathrm{~cm}$ viewing distance and were performed in order from low spatial frequency to high. The first presentation, with low spatial frequency (low-SF) of 0.3 cycles per degree (cpd), is unlikely to induce any distortions or discomforts and is designed to ensure subjects provide true answers; The second pattern is presented with a mid-spatial frequency (mid-SF) gratings of $2.3 \mathrm{cpd}$ and is able to induce maximum visual distortions in susceptible subjects; The third one with a higher spatial frequency (high-SF) of $9.4 \mathrm{cpd}$ is expected to induce fewer symptoms than the second pattern. For every pattern, participants were instructed to fixated on the central of the presentation for $5 \mathrm{~s}$, after which they were asked (yes or no) whether they experienced the following 15 symptoms: red, green, blue, yellow, bending of lines, blurring of lines, shimmering of lines, flickering, fading, shadowy shapes among the lines, pain, discomfort, nausea, dizziness, unease [50]. The numbers of the yes responses to the above symptoms were summed to generate a pattern glare score for each of the gratings. We also obtained an indirect score by subtracting the score of high-SF grating from the score of the mid-SF grating, namely mid-high difference variable.

\section{Statistical analysis}

We used SPSS 25.0 software (IBM Corporation, Armonk, NY, USA) for Win10 to analyze all the data with a significance level of $p=0.05$ used throughout the analysis. Continuous variables were presented as mean $(\mathrm{M})$ and standard deviation (SD) and relative ratio was used to describe categorical variable. Comparisons of demographic characteristics and pattern glare scores between MDDs group and HCs were performed. The MDDs group was then divided into first episode medication-free group (fMDD) and recurrent medicated group (rMDD) to compare the pattern glare scores between them. Before the statistical analysis of the data, the quantitative variables were checked for normal distribution. Quantitative variables normally distributed were compared using two-sample $t$ test, while those non-normally distributed were analyzed using MannWhitney U test. Categorical variable was compared using chi-square test. Spearman and Pearson analysis were utilized to detect whether there exist correlations between some indexes (i.e. age, HAMD score and illness duration) and pattern glare scores in both MDDs and $\mathrm{HCs}$ group. ROC (receiver operating characteristic) curve was used to evaluate whether pattern glare test was able to identify patients and healthy controls. Full details of data analyses were given in additional files.

\section{Results}

\section{Demographic characteristics}

Demographic data presented that MDDs group and HCs group were well matched for age $(\mathrm{M} \pm \mathrm{SD}$; MDD: $30.50 \pm$ 10.714; HCs: $27.70 \pm 6.287 ; P=0.265$ ), gender distribution (male/female; MDDs: 9/15; HCs: $14 / 16 ; P=0.498$ ) and education years $(\mathrm{M} \pm \mathrm{SD}$; $\mathrm{MDD}$ : $14.92 \pm 2.412$; HCs: $16.00 \pm 2.421 ; P=0.108)$. The 24 MDDs exhibited a HAMD score of $22.21 \pm 4.334(\mathrm{M} \pm \mathrm{SD})$, indicating that the patients were going through a depressive episode by the time of entering the study. The basic characteristics of the two groups were shown in Table 1.

$P^{a}=$ Chi-square test; $P^{b}=$ two-sample $\mathrm{t}$ test; HAMD = Hamilton Depression Rating Scale; Descriptive variables were described with mean \pm standard deviation; Gender was described with relative ratio; $P<0.05$ was considered as statistically significant.

\section{Differences of pattern glare scores between MDDs and HCs}

The two groups differed significantly in pattern glare score of mid-SF grating (two-sample t test: $P=0.023$ ), mid-SF score being significantly higher in MDDs group than the in HCs group. There were no statistical differences in pattern glare scores of low-SF grating (Mann-Whitney $U$ test: $P=0.485$ ), high-SF grating (Mann-Whitney $U$ test: $P=0.092$ ) and the mid-high difference (two-sample t test: $P=0.731$ ) between the two groups (Table 2). The result indicated elevated level

Table 1 Demographic and clinical data of MDDs and HCs groups

\begin{tabular}{llll}
\hline Variables & MDDs & HCs & $P$ value \\
\hline Age (years) & $30.50 \pm 10.714$ & $27.70 \pm 6.287$ & $0.265^{b}$ \\
Gender (male/female) & $9 / 15$ & $14 / 16$ & $0.498^{a}$ \\
Education level (years) & $14.92 \pm 2.412$ & $16.00 \pm 2.421$ & $0.108^{b}$ \\
HAMD score & $22.21 \pm 4.334$ & - & - \\
Illness duration (months) & $42.21 \pm 56.628$ & - & - \\
\hline
\end{tabular}


of pattern glare was induced by the mid-SF pattern rather than the other two gratings.

Data were described with mean \pm standard deviation; $P<0.05$ was deemed to be statistically significant.

\section{Comparisons of PGS between $\mathrm{FMDD}$ and $\mathrm{rMDD}$}

No statistical differences in pattern glare scores between the first episode medication-free group (fMDD) and recurrent medication undergoing group (rMDD) were found (Table 3).

$\mathrm{fMDD}=$ first episode medication-free patients with MDD; $r M D D=$ recurrent medication-ongoing patients with MDD; Data were described with mean \pm standard deviation; $P<0.05$ was considered to be statistically significant.

\section{Correlations between pattern glare scores and some clinical indexes}

Of all the correlation analysis, there was a significant negative correlation between mid-high difference and age in HCs group only (Spearman correlation analysis: $r=-0.421, P=0.021$ ); no any other significant correlations were found between pattern glare scores and all the other variables (Table 4 ).

$$
P<0.05 \text { was considered to be statistically significant. }
$$

\section{Clinical diagnostic value of mid-SF pattern glare score in major depressive disorder}

Since the mid-SF pattern glare score exhibited significant difference between MDDs group and HCs group, we conducted ROC analysis and found the area under the curve of mid-SF scores was 0.668 ( $p=0.035$, Fig. 1), indicating the mid-SF has limited value to discriminate MDDs from HCs.

\section{Discussion}

We demonstrated that there exists high level of pattern glare in major depressive disorder. The MDDs group scored higher in the mid and high frequencies but not low frequency pattern gratings than the HCs group. Previous studies have found that specifically the mid-SF pattern that would induce most distortions. The mid-SF score and mid-high difference are the two most distinctive indications of pattern glare [23, 36, 43]. Significantly negative correlation between mid-high difference and age in HCs group was found. Our performance of ROC

Table 2 Comparisons of PGS between MDDs and HCs

\begin{tabular}{llll}
\hline Variables & MDDs & HCs & $P$ value \\
\hline PGS of low-SF(p1) & $1.00 \pm 1.668$ & $0.57 \pm 0.935$ & 0.485 \\
PGS of mid-SF(p2) & $4.58 \pm 2.552$ & $3.13 \pm 1.776$ & 0.023 \\
PGS of high-SF(p3) & $3.42 \pm 2.669$ & $2.20 \pm 1.937$ & 0.092 \\
mid-high SF difference(s) & $1.17 \pm 1.949$ & $0.97 \pm 2.236$ & 0.731 \\
\hline
\end{tabular}

Table 3 Comparisons of PGS between fMDD and rMDD

\begin{tabular}{llll}
\hline Variables & fMDD & rMDD & $P$ value \\
\hline PGS of low-SF(p1) & $1.70 \pm 2.214$ & $0.50 \pm 0.941$ & 0.094 \\
PGS of mid-SF(p2) & $5.10 \pm 2.283$ & $4.21 \pm 2.751$ & 0.414 \\
PGS of high-SF(p3) & $3.30 \pm 3.164$ & $3.50 \pm 2.378$ & 0.861 \\
mid-high SF difference(s) & $1.80 \pm 1.874$ & $0.71 \pm 1.939$ & 0.201 \\
\hline
\end{tabular}

analysis using pattern glare score of mid-SF showed the score possesses limited value of identifying the depressed and the healthy.

Several lines of evidences reported decreased GABA levels in occipital cortex, anterior cingulate cortex (a prefrontal cortical region) and the dorsomedial/dorsal anterolateral prefrontal ROI in MDD subjects and even in recovered depressed patients [51-55].

The association between altered GABA levels and vision abnormity has also been reported frequently. Visual deficit-poor vision-was found to be rendered by loss of dendritic cell factor 1 through the GABA system in mouse primary visual cortex [56]. In the early stage of type 2 diabetes, occipital cortical GABA has been reported to be a novel predictor of visual psychophysical performance, that is, speed and achromatic discrimination thresholds [57]. The application of GABA and its agonist to senescent macaques lead to improved orientation and direction, an enhanced ability to signal visual stimuli, combined with decreased visual responsiveness and spontaneous activity [58]. Increased visual cortical GABA levels was found to be correlated with longer percept durations [59]. Remarkably reduced occipital GABA concentrations has been reported to be possibly responsible for the visual problem in first-episode, unmedicated MDD [60].

These findings thrown some light on interpreting pattern glare via the GABA system. The symptoms of pattern glare are more obvious under binocular than monocular conditions, GABA being the neurotransmitter whose interneurons are responsible for the combination of the input from the two eyes in the cortex, which suggest a role of GABA in the mechanism of pattern glare [24, 27, 61]. Additionally, the decreased GABA levels in MDD lead to deficient cortical inhibition or cortical hyper-excitability and thus high level of pattern glare, cortical hyper-excitability being deemed to be the mechanism of pattern glare.

Whether psychiatric stress or other psychiatric conditions, such as anxiety or schizophrenia, plays a role in increasing pattern glare score could be concerned. The patients in our study were free of other psychiatric symptoms. It is well worth involving more psychiatric diseases, such as anxiety disorder, schizophrenia and obsessive-compulsive disorder, in the future study of pattern glare. 
Table 4 Correlations between pattern glare scores and several clinical indexes

\begin{tabular}{|c|c|c|c|c|}
\hline & Group & Age & HAMD score & Illness duration \\
\hline \multirow[t]{4}{*}{ PGS of low-SF gratings ( $\mathrm{p} 1$ ) } & MDDs & $r=-0.181$ & $r=-0.163$ & $r=-0.049$ \\
\hline & & $p=0.397$ & $p=0.447$ & $p=0.821$ \\
\hline & $\mathrm{HCs}$ & $r=0.008$ & & \\
\hline & & $p=0.968$ & & \\
\hline \multirow[t]{4}{*}{ PGS of mid-SF ratings (p2) } & MDDs & $r=-0.277$ & $r=0.079$ & $r=-0.337$ \\
\hline & & $p=0.190$ & $p=0.714$ & $p=0.107$ \\
\hline & $\mathrm{HCs}$ & $r=-0.263$ & & \\
\hline & & $p=0.161$ & & \\
\hline \multirow[t]{4}{*}{ PGS of high-SF gratings(p3) } & MDDs & $r=-0.154$ & $r=0.126$ & $r=-0.148$ \\
\hline & & $p=0.474$ & $p=0.556$ & $p=0.491$ \\
\hline & $\mathrm{HCs}$ & $r=0.164$ & & \\
\hline & & $p=0.388$ & & \\
\hline \multirow[t]{4}{*}{ Scores of mid-high SF(s) } & MDDs & $r=-0.184$ & $r=0.032$ & $r=-0.252$ \\
\hline & & $p=0.390$ & $p=0.883$ & $p=0.235$ \\
\hline & $\mathrm{HCs}$ & $r=-0.421$ & & \\
\hline & & $p=0.021$ & & \\
\hline
\end{tabular}

The result of significantly negative correlation between mid-high SF difference and age in HCs group showed an overall decreasing level of pattern glare with age, which is consistent with previous finding that there existed a significant inverse correlation between age and the pattern glare score for the $3 \mathrm{cpd}$. The result indicated increased cortical inhibition with age, agreeing with a recent finding that visual cortical GABA levels increased in older adults [59]. Primary cortex synchrony,

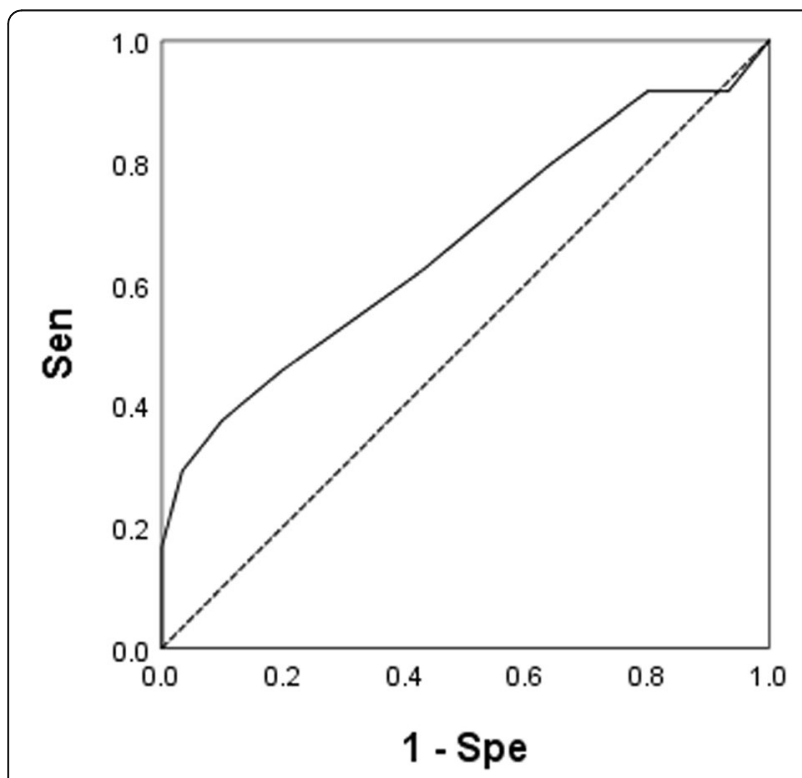

Diagonal segments are produced by ties.

Fig. 1 ROC curve of ' $p 2$ '. AUC: 0.668 (95\% Cl:0.519-0.817), $p=0.035$ contributing to cortical hyperexcitability, was reported to get losing with age in monkey, which leads to a decreasing level of pattern glare with age [62, 63].

Many optical changes in the eye with age including lens changes, increased aberrations and senile pupillary miosis can result in a reduction of the contrast sensitivity function; these changes may give rise to lower level of light reaching the retina and might have an influence on the results of the pattern glare test with age $[27,64]$. The loss of sensory acuity with age may also be responsible for the decreased pattern glare scores [27]. However, the MDDs group displayed no negative correlation between age and pattern glare scores. Since the age between the MDDs and the HCs did not differ significantly, this result indicated relatively reduced cortical inhibition in MDDs group compared to the HCs group, which is consistent to our assumption.

The ROC analysis of pattern 2 displayed limited clinical diagnostic value of identifying the depressed and the healthy, the area under the curve being not so large. But we hold that the test deserves further exploration, for it is rather easy to implement, but may represent a new potential biomarker of diagnosing MDD. Replication of the test with large sample size is warranted to explore whether it is possible to find the diagnostic value of pattern 2 more powerful.

Our study has two limitations. First, though there were no significant differences in pattern glare score between first episode mediation-free subjects and recurrent medication-ongoing patients, it is unclear whether the effect of disease episode numbers on pattern glare scores and the effect of medicine can influence each other. 
Three groups, including healthy controls, first-episode medication-free subjects and the subjects after medication therapy can be set to explore the effects of medicine on pattern glare score in future study. Second, our study was limited by a small sample size. The results need to be verified in future study for it is the first time the pattern glare test has been applied to major depressive disorder.

\section{Conclusions}

In conclusion, we feel that our study is valuable, for it is a rather simple test with a promise of being a diagnostic tool of major depressive disorder. We mainly found the score difference in mid-SF of $2.3 \mathrm{cpd}$ gratings between MDDs and HCs group was significant and the pattern 2 has limited value in the diagnosis of major depressive disorder. Since the study is the first one applying the pattern glare test in MDD, well-designed replication is needed to further verify the outcome.

\section{Abbreviations}

HAMD: Hamilton Depression Rating Scale; HC: Healthy control; MDD: Major depressive disorder; PGS: Pattern glare score; ROC: Receiver operating characteristic

\section{Acknowledgements}

The authors thank all individuals who have participated in this study.

\section{Authors' contributions}

All authors have made significant scientific contributions to this manuscript. X.H.M., E.C. and T.L. conceived and designed the experiments. X.W.Q., H.H.F., X.Y., and Y.Y.C. performed the experiments. X.W.Q., H.H.F., X.Y., X.H.M., W.J.G., Q.W. and W.D. analyzed the data. X.W.Q., H.H.F., and X.H.M. wrote the manuscript. All authors reviewed the manuscript. All authors read and approved the final manuscript.

\section{Funding}

This research was partly funded by National Natural Science Foundation of China (Grant No. 81671344). The funding body had no role in the design of the study and collection, analysis, and interpretation of data and in writing the manuscript.

\section{Availability of data and materials}

The datasets used and analyzed during the current study are not publicly available due to no permission of the ethics committee, but are available from the corresponding author on reasonable request.

\section{Ethics approval and consent to participate}

The study was approved by the West China Hospital of Sichuan University ethics committee. All the 54 subjects provided written informed consent.

\section{Consent for publication}

Not applicable.

\section{Competing interests}

The authors declare that they have no competing interests.

\section{Author details}

${ }^{1}$ Psychiatric Laboratory and Mental Health Center, West China Hospital of Sichuan University, Chengdu, Sichuan 610064, People's Republic of China. ${ }^{2}$ West China Brain Research Center, West China Hospital of Sichuan University, Chengdu, China. ${ }^{3}$ Department of Psychiatry, The University of Hong Kong, Queen Mary Hospital, Pokfulam, Hong Kong.
Received: 22 May 2019 Accepted: 9 December 2019

Published online: 21 December 2019

\section{References}

1. Smith K. Mental health: a world of depression. Nature. 2014;515(7526):181.

2. Otte C, Gold SM, Penninx BW, Pariante CM, Etkin A, Fava M, et al. Major depressive disorder. Nat Rev Dis Primers. 2016;2:16065.

3. Jat Ml, Afridi MI, Amar W, Lal C. Prevalence of migraine among patients of depressive disorder. Pak J Med Sci. 2018;34(4):964-7.

4. Lee SM, Yoon JR, Yi YY, Eom S, Lee JS, Kim HD, et al. Screening for depression and anxiety disorder in children with headache. Korean J Pediatr. 2015;58(2):64-8.

5. Muneer A, Farooq A, Faroog JH, Qurashi MS, Kiani IA, Farooq JS. Frequency of primary headache syndromes in patients with a major depressive disorder. Cureus. 2018;10(6):e2747.

6. Scanlon GC, Jain FA, Hunter AM, Cook IA, Leuchter AF. Neurophysiologic correlates of headache pain in subjects with major depressive disorder. Clin EEG Neurosci. 2017;48(3):159-67.

7. Zhao D, Wu Z, Zhang H, Mellor D, Ding L, Wu H, et al. Somatic symptoms vary in major depressive disorder in China. Compr Psychiatry. 2018;87:32-7.

8. GBD 2016 Disease and Injury Incidence and Prevalence Collaborators. Global, regional, and national incidence, prevalence, and years lived with disability for 328 diseases and injuries for 195 countries, 1990-2016: a systematic analysis for the Global Burden of Disease Study 2016. Lancet. 2017;390(10100):1211-59.

9. Anagnostou E, Vikelis M, Tzavellas E, Ghika A, Kouzi I, Evdokimidis I, et al. Photophobia in primary headaches, in essential blepharospasm and in major depression. Int J Neurosci. 2017;127(8):673-9.

10. Chang $Y, X u$ J, Shi N, Pang X, Zhang B, Cai Z. Dysfunction of preattentive visual information processing among patients with major depressive disorder. Biol Psychiatry. 2011;69(8):742-7.

11. Friberg TR, Borrero G. Diminished perception of ambient light: a symptom of clinical depression? J Affect Disord. 2000;61(1-2):113-8.

12. Friberg TR, Bremer RW, Dickinsen M. Diminished perception of light as a symptom of depression: further studies. J Affect Disord. 2008;108(3):235-40.

13. Qiu X, Yang X, Qiao Z, Wang L, Ning N, Shi J, et al. Impairment in processing visual information at the pre-attentive stage in patients with a major depressive disorder: a visual mismatch negativity study. Neurosci Lett. 2011;491(1):53-7.

14. Zhang X, Bullard KM, Cotch MF, Wilson MR, Rovner BW, McGwin G Jr, et al. Association between depression and functional vision loss in persons 20 years of age or older in the United States, NHANES 2005-2008. JAMA ophthalmology. 2013;131(5):573-81.

15. Chen C, Jiang WH, Wang W, Ma XC, Li Y, Wu J, et al. Impaired visual, working, and verbal memory in first-episode, drug-naive patients with major depressive disorder in a Chinese population. PLoS One. 2018;13(4):e0196023.

16. Hammar A, Lund A, Hugdahl K. Selective impairment in effortful information processing in major depression. J Int Neuropsychol Soc. 2003;9(6):954-9.

17. Bubl E, Kern E, Ebert D, Bach M. Tebartz van Elst L. seeing gray when feeling blue? Depression can be measured in the eye of the diseased. Biol Psychiatry. 2010;68(2):205-8.

18. Bubl E. Tebartz Van Elst L, Gondan M, Ebert D, Greenlee MW. Vision in depressive disorder. World J Biol Psychiatry. 2009;10(4 Pt 2):377-84.

19. Fam J, Rush AJ, Haaland B, Barbier S, Luu C. Visual contrast sensitivity in major depressive disorder. J Psychosom Res. 2013;75(1):83-6.

20. Normann C, Schmitz D, Furmaier A, Doing C, Bach M. Long-term plasticity of visually evoked potentials in humans is altered in major depression. Biol Psychiatry. 2007:62(5):373-80.

21. Bubl E, Ebert D, Kern E, van Elst LT, Bach M. Effect of antidepressive therapy on retinal contrast processing in depressive disorder. Br J Psychiatry. 2012; 201:151-8.

22. London A, Benhar I, Schwartz M. The retina as a window to the brain-from eye research to CNS disorders. Nat Rev Neurol. 2013;9(1):44-53.

23. Monger $L$, Wilkins AJ, Allen PM. Pattern glare: the effects of contrast and color. Front Psychol. 2015;6:1651.

24. Wilkins A, Nimmo-Smith I. On the reduction of eye-strain when reading. Ophthalmic Physiol Opt. 1984;4(1):53-9.

25. Wilkins A, Nimmo-Smith I, Tait A, McManus C, Della Sala S, Tilley A, et al. A neurological basis for visual discomfort. Brain. 1984;107(Pt 4):989-1017.

26. Evans B, Drasdo N. Tinted lenses and related therapies for learning disabilities--a review. Ophthalmic Physiol Opt. 1991;11(3):206-17. 
27. Evans BJ, Stevenson SJ. The pattern glare test: a review and determination of normative values. Ophthalmic Physiol Opt. 2008;28(4):295-309.

28. Braithwaite J, Mevorach C, Takahashi C. Stimulating the aberrant brain: evidence for increased cortical hyperexcitability from a transcranial direct current stimulation (tDCS) study of individuals predisposed to anomalous perceptions. Cortex. 2015;69:1-13.

29. Evans BJ, Allen PM. A systematic review of controlled trials on visual stress using intuitive overlays or the intuitive colorimeter. Aust J Optom. 2016;9(4): 205-18.

30. Haigh S, Cooper N, Wilkins A. Cortical excitability and the shape of the haemodynamic response. Neuroimage. 2015;111:379-84.

31. Haigh SM, Barningham L, Berntsen M, Coutts LV, Hobbs ES, Irabor J, et al. Discomfort and the cortical haemodynamic response to coloured gratings. Vis Res. 2013:89:47-53.

32. Han D, Wegrzyn J, Bi H, Wei R, Zhang B, Li X. Practice makes the deficiency of global motion detection in people with pattern-related visual stress more apparent. PLoS One. 2018;13(2):e0193215.

33. Huang J, Zong X, Wilkins A, Jenkins B, Bozoki A, Cao Y. fMRI evidence that precision ophthalmic tints reduce cortical hyperactivation in migraine. Cephalalgia. 2011;31(8):925-36.

34. Huang J, Cooper TG, Satana B, Kaufman DI, Cao Y. Visual distortion provoked by a stimulus in migraine associated with hyperneuronal activity. Headache. 2003;43(6):664-71.

35. Braithwaite JJ, Broglia E, Brincat O, Stapley L, Wilkins AJ, Takahashi C. Signs of increased cortical hyperexcitability selectively associated with spontaneous anomalous bodily experiences in a nonclinical population. Cogn Neuropsychiatry. 2013;18(6):549-73.

36. Beasley IG, Davies LN. Susceptibility to pattern glare following stroke. J Neurol. 2012;259(9):1832-9.

37. Harle DE, Evans BJ. The optometric correlates of migraine. Ophthalmic Physiol Opt. 2004;24(5):369-83.

38. Harle DE, Shepherd AJ, Evans BJ. Visual stimuli are common triggers of migraine and are associated with pattern glare. Headache. 2006;46(9):1431-40.

39. Newman Wright B, Wilkins A, Zoukos Y. Spectral filters can improve reading and visual search in patients with multiple sclerosis. J Neurol. 2007;254(12): $1729-35$.

40. Robertson AE, David RSR. The sensory experiences of adults with autism spectrum disorder: a qualitative analysis. Perception. 2015;44(5):569-86.

41. Ward J, Hoadley C, Hughes JE, Smith P, Allison C, Baron-Cohen S, et al. Atypical sensory sensitivity as a shared feature between synaesthesia and autism. Sci Rep. 2017;7:41155.

42. Wilkins A, Huang J, Cao Y. Visual stress theory and its application to reading and reading tests. J Res Read. 2004;27(2):152-62.

43. Wilson RL, Paterson KB, Hutchinson CV. Increased vulnerability to patternrelated visual stress in Myalgic encephalomyelitis. Perception. 2015;44(12): 1422-6.

44. Hou Z, Jiang W, Yin Y, Zhang Z, Yuan Y. The current situation on major depressive disorder in China: research on mechanisms and clinical practice. Neurosci Bull. 2016;32(4):389-97.

45. First MB, Spitzer RL, Miriam G, Williams JBW. User's guide for the structured clinical interview for DSM-IV axis I disorders : SCID-I : clinical version: American psychiatric press; 1997.

46. Stone P, Kaminka GA, Rosenschein JS, Dsmiv TFO, Association AP. Diagnostic and statistical manual of mental disorders: DSM-IV-TR. Encyclopedia Neurol Sci. 2000;25(2):4-8.

47. Hetherington R. The Snellen chart as a test of visual acuity. Psychol Forsch. 1954;24(4):349-57.

48. Peters HB. Vision screening with a Snellen chart. Am J Optom Arch Am Acad Optom. 1961;38:487-505.

49. Rhiu S, Lee HJ, Goo YS, Cho K, Kim JH. Visual acuity testing using a random method visual acuity application. Telemed J E Health. 2016;22(3):232-7.

50. Allen PM, Hussain A, Usherwood C, Wilkins AJ. Pattern-related visual stress, chromaticity, and accommodation. Invest Ophthalmol Vis Sci. 2010;51(12): 6843-9.

51. Bhagwagar Z, Wylezinska M, Jezzard P, Evans J, Ashworth F, Sule A, et al. Reduction in occipital cortex gamma-aminobutyric acid concentrations in medication-free recovered unipolar depressed and bipolar subjects. Biol Psychiatry. 2007;61(6):806-12

52. Gabbay V, Bradley KA, Mao X, Ostrover R, Kang G, Shungu DC. Anterior cingulate cortex gamma-aminobutyric acid deficits in youth with depression. Transl Psychiatry. 2017;7(8):e1216.
53. Hasler G, van der Veen JW, Tumonis T, Meyers N, Shen J, Drevets WC. Reduced prefrontal glutamate/glutamine and gamma-aminobutyric acid levels in major depression determined using proton magnetic resonance spectroscopy. Arch Gen Psychiatry. 2007;64(2):193-200.

54. Price RB, Shungu DC, Mao X, Nestadt P, Kelly C, Collins KA, et al. Amino acid neurotransmitters assessed by proton magnetic resonance spectroscopy: relationship to treatment resistance in major depressive disorder. Biol Psychiatry. 2009;65(9):792-800.

55. Salari AA, Bakhtiari A, Homberg JR. Activation of GABA-A receptors during postnatal brain development increases anxiety- and depression-related behaviors in a time- and dose-dependent manner in adult mice. Eur Neuropsychopharmacol. 2015;25(8):1260-74.

56. Shi J, Li Q, Wen T. Dendritic cell factor 1-knockout results in visual deficit through the GABA system in mouse primary visual cortex. Neurosci Bull. 2018;34(3):465-75

57. Sanches M, Abuhaiba SI, d'Almeida OC, Quendera B, Gomes L, Moreno C, et al. Diabetic brain or retina? Visual psychophysical performance in diabetic patients in relation to GABA levels in occipital cortex. Metab Brain Dis. 2017; 32(3):913-21.

58. Leventhal AG, Wang Y, Pu M, Zhou Y, Ma Y. GABA and its agonists improved visual cortical function in senescent monkeys. Science. 2003; 300(5620):812-5.

59. Pitchaimuthu K, Wu QZ, Carter O, Nguyen BN, Ahn S, Egan GF, et al. Occipital GABA levels in older adults and their relationship to visual perceptual suppression. Sci Rep. 2017;7(1):14231.

60. Song Z, Huang P, Qiu L, Wu Q, Gong Q, Zhang B, et al. Decreased occipital GABA concentrations in patients with first-episode major depressive disorder: a magnetic resonance spectroscopy study. Sheng Wu Yi Xue Gong Cheng Xue Za Zhi. 2012;29(2):233-6.

61. Beristain X. Essentials of neural science and behavior : By Eric R. Kandel, James H. Schwartz, and Thomas M. Jessell743 pages. \$47.95.Norwalk Ct: Appleton \& Lange, 1995.ISBN 0-8385-2245-9. Surg Neurol. 1996;45(2):206.

62. Peters $A$, Moss $M B$, Sethares $C$. Effects of aging on myelinated nerve fibers in monkey primary visual cortex. J Comp Neurol. 2000;419(3):364-76.

63. Wilkins A. Photosensitive Epilepsy and Visual Discomfort; 1989.

64. Owsley C, Gardner T, Sekuler R, Lieberman H. Role of the crystalline lens in the spatial vision loss of the elderly. Invest Ophthalmol Vis Sci. 1985;26(8): $1165-70$.

\section{Publisher's Note}

Springer Nature remains neutral with regard to jurisdictional claims in published maps and institutional affiliations.
Ready to submit your research? Choose BMC and benefit from:

- fast, convenient online submission

- thorough peer review by experienced researchers in your field

- rapid publication on acceptance

- support for research data, including large and complex data types

- gold Open Access which fosters wider collaboration and increased citations

- maximum visibility for your research: over $100 \mathrm{M}$ website views per year

At BMC, research is always in progress.

Learn more biomedcentral.com/submissions 